\title{
Evidence of integrated primary-secondary health care in low-and middle-income countries: protocol for a scoping review
}

\author{
Md Zabir Hasan ${ }^{1 *}$ D, Shalini Singh ${ }^{1}$, Dinesh Arora', Nishant Jain² and Shivam Gupta ${ }^{1}$
}

\begin{abstract}
Background: Integrated care is a people-centered health delivery approach that ensures the comprehensiveness, quality, and continuity of service across the settings and levels of health systems. The World Health Organization (WHO) recommends integration across levels and building-blocks of health systems as a prerequisite of Universal Health Coverage (UHC). While health systems of low- and middle-income countries (LMICs) are often fragmented and led by siloed service delivery structure, several LMICs —including India—have attempted health system integration. Several systematic reviews of evidence on healthcare integration from developed countries exist, but no synthesis from LMICS was reported to date. This review will overview the existing evidence of primary-secondary care integration (PSI) in the context of LMICs, aiming to support policy decisions for the effective integration of health delivery systems in India.

Methods: The review will be conducted following the six steps recommend by Arksey and O'Malley. Scientific and grey literature will be systematically selected from PubMed, Embase, Scopus, Web of Science, Global Index Medicus, and electronic repositories (such as WHO, World Bank, Health Policy Plus, and OpenGrey). Using a comprehensive search strategy, literature written in English and published between 2000 and 2020 will be selected, and two independent authors will screen their titles and abstracts. The result will be charted using a data extraction form and reported using tables, figures, and narrative forms.

Discussion: No ethical approval is necessary for the review. The final report will be developed with the consultation of other stakeholders and disseminated through workshops, conference papers, and peer review articles. The review will serve as a guiding tool to approach, implement, and test the PSI models in India and other LMICS.
\end{abstract}

Scoping review registration: https://osf.io/kjhzt.

Keywords: Integrated care, Integrated primary-secondary care, Health systems, Ayushman Bharat, Scoping review, Low- and middle-income countries, India

\footnotetext{
* Correspondence: zabir.hasan@gmail.com; mhasan10@jhu.edu

'Department of International Health, Johns Hopkins Bloomberg School of Public Health, Baltimore, MD, USA

Full list of author information is available at the end of the article
}

(c) The Author(s). 2020 Open Access This article is licensed under a Creative Commons Attribution 4.0 International License, which permits use, sharing, adaptation, distribution and reproduction in any medium or format, as long as you give appropriate credit to the original author(s) and the source, provide a link to the Creative Commons licence, and indicate if changes were made. The images or other third party material in this article are included in the article's Creative Commons licence, unless indicated otherwise in a credit line to the material. If material is not included in the article's Creative Commons licence and your intended use is not permitted by statutory regulation or exceeds the permitted use, you will need to obtain permission directly from the copyright holder. To view a copy of this licence, visit http://creativecommons.org/licenses/by/4.0/ The Creative Commons Public Domain Dedication waiver (http://creativecommons.org/publicdomain/zero/1.0/) applies to the data made available in this article, unless otherwise stated in a credit line to the data. 


\section{Background}

\section{Integrated primary-secondary care system}

Health service integration is seen by the World Health Organization (WHO) as an essential requirement to achieve Universal Health Coverage (UHC) [1]. According to $\mathrm{WHO}$, an integrated health service delivery is defined as:

... an approach to strengthen people-centered health systems through the promotion of the comprehensive delivery of quality services across the life-course, designed according to the multidimensional needs of the population and the individual and delivered by a coordinated multidisciplinary team of providers working across settings and levels of care... ... with feedback loops to continuously improve performance and to tackle upstream causes of ill health and to promote well-being through intersectoral and multisectoral actions [2].

The definition itself reveals the multidimensionality of the integrated care system. As Fig. 1 indicates, integration can happen across the level of care (such as primary, secondary, or tertiary), between the building blocks of the health systems (service delivery, human resource, medicine and technologies, financing, health information, or governance) and even across geography or time-span [3]. Moreover, based on its nature, integrated care can be divided into (a) Organizational: different health service organizations are merged for care coordination; (b) Functional: non-clinical services are related with integration (such as administration, electrical supply, etc.); (c) Service: different type or genre of service merged at facility level using a multi-disciplinary team; and lastly (d) Clinical: application of shared guideline and protocol to treat comorbidity or ensuring continuum of care $[4,5]$.
The primary-secondary care integration (PSI) is a part of vertical integration where the primary healthcare system is efficiently connected with the rest of the healthcare delivery structure, but most importantly, with secondary care. Within the hierarchical structure of the health system, PSI can bring "closer-to-community services" within broader management (organizational integration) or provide the opportunity for functional, clinical, or service level integration [4]. It opens the prospect for organized service delivery between primary care provider and specialist, leading to early detection of disease, improved quality of care, better follow-up, and patient outcome [6-10]-however-effectiveness of the integration can vary by context and interventions [11].

\section{Indian health systems in transition}

While high-income countries are already moving to integrated service configurations and delivery structure, lowand middle-income countries (LMICs) have yet to significantly implement integrated care in their public health systems, mostly because of the complexities of implementation in a context where healthcare infrastructure and human resources for health are inadequate [1]. For example, the Government of India launched the Ayushman Bharat (AB) program in 2018 to restructure the public financing for healthcare and service delivery mechanisms toward UHC [12].

The AB program consists of two unique-but complementary-components: (a) the Pradhan Mantri Jan Arogya Yojana (PM-JAY) and (b) scaling up Health and Wellness Centres (HWCs) [12]. PM-JAY is considered the largest health assurance program globally, providing financial protection for 500 million of the poorest and vulnerable individuals [13]. Under this scheme, a family will be covered up to 500,000 Rupee (approximately 6978 USD, considering the exchange rate of 71.65 Rupee $=1$ USD on 2 January 2020) per

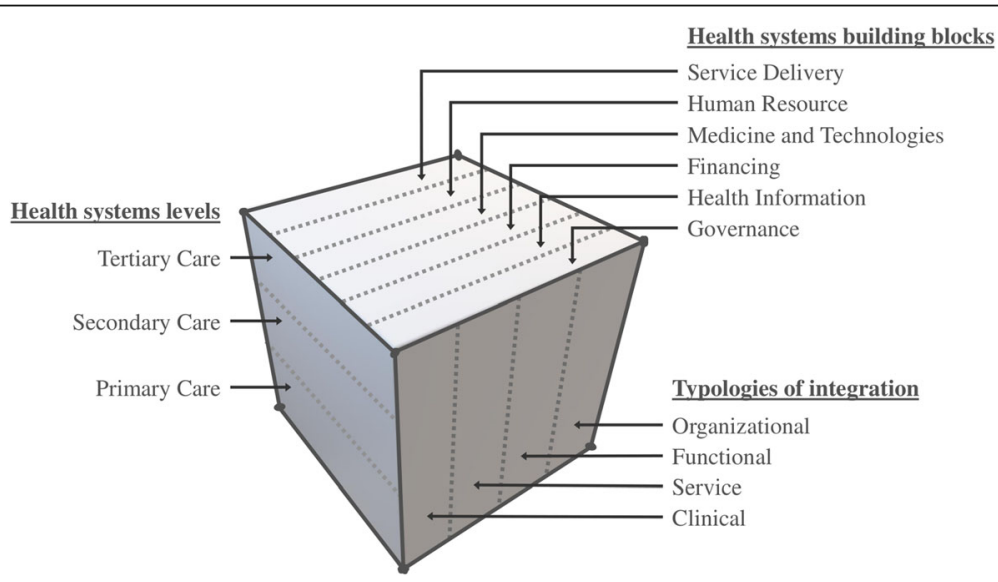

Fig. 1 Complexity of vertical integration within health systems 
year. This will cover approximately 1393 procedures, including doctor's fees, drugs, supplies, diagnostics, room charges, etc. However, these benefits are eligible for only secondary and tertiary care services [14].

While implementing PM-JAY, the national and state health systems of India are transitioning through several structural integrations. PM-JAY is administered by a separate National Health Authority (NHA) at the national level. However, the individual state retains the flexibility to implement the scheme through the State Health Agency (SHA). State governments must contribute $40 \%$ of the cost of running the program and are encouraged to merge state-funded insurance schemes with PM-JAY to expand the risk pool. All public secondary and tertiary hospitals are automatically included under PM-JAY. The private health sector has been integrated within PM-JAY by empaneling private hospitals based on defined criteria linked to specific service packages [15]. Further, functional integrations are also evident within the PM-JAY scheme through the portability of services across states.

While implementing the $\mathrm{AB}$ program, the Government of India is establishing 150,000 HWCs to prepare the primary healthcare delivery system for the emerging epidemiologic transition [16]. HWCs are located within 30 min, traveling distance from any community. HWCs will provide an expanded range of services that will be available, including reproductive, maternal, and child health, preventive and curative care for communicable and chronic diseases, and support for mental health, etc. [16]. To make it as the first point of contact closer to the community, existing rural clinics-designated as sub-centers-and rural and urban primary health centers (PHCs) will be upgraded as HWCs to deliver comprehensive primary health care (CPHC) [17].

Since its launch till February 2020, PM-JAY has empaneled over 21 thousand hospitals, issued more than 12 million beneficiary cards, and provided financial support for 3.6 million beneficiaries [13, 14]. Simultaneously, over 30 thousand HWCs have been established by transforming 13,516 Sub-centers, 13,417 PHCs, and 3076 urban PHCs [16]. However, the critical link between primary-secondary care provision is still missing, as PMJAY does not yet provide financial support at these primary level facilities. Moreover, the care continuum pathway is still evolving beyond HWCs, owing to its early implementation stage.

\section{Rationale of the review}

The AB program's progress has been promising so far, yet some critical design challenges have been identified. The fact that PM-JAY will not cover primary care, it is even more imperative to plan strategies under $A B$ to increase the accountability of HWCs to deliver CPHC so that people do not bypass preventive and promotive services offered by HWCs. This will limit efforts of secondary prevention and lead to increased care-seeking from secondary or tertiary care facilities. As the demand for service will increase, the overburdened secondary or tertiary care facilities will face issues with the quality of care. More importantly, increasing demand will jeopardize the fiscal sustainability of PM-JAY [18].

Thus, the role of HWCs is paramount in this case. As the first point of contact, HWCs are mandated to keep the population healthy through prevention strategies and extensive lifestyle counseling. This will contribute to a lower frequency of procedures or curative care events. Further strengthening of HWCs by focusing on the continuum of care-starting from early initiation of preventive care, judicious use of curative care, proper referral, follow up care, and educating the beneficiaries about PM-JAY - need to be prioritized [19]. This calls for effective integration of primary and secondary care between HWCs and other secondary-level public and private providers within the $\mathrm{AB}$ program.

Therefore, to enable implementation of PSI models within the $A B$ system, there is a need to identify design features that can be adopted within the healthcare delivery systems as part of $A B$ to enable a sound linkage across different service provision levels. Other existing structural deficiencies of the Indian health care delivery system-such as governance, stewardship, quality of care, and health system organization-will also impose additional layers of complexity. Thus, looking at the global best practices and experience of health service integration is the most pragmatic way forward.

Several systemic and scoping reviews were already conducted exploring the PSI models; however, the majority of those studies were conducted in the context of developed countries [7, 8, 20-22]. Some evidence from LMICs has already shown that vertical integration of primary and secondary care has a strong potential to increase access, reduce cost, and improve quality and health outcomes [21, 22]. There is an insufficient exploration of critical aspects of design, implementation of strategies, assessment of PSI models, and consolidation of evidence from other LMICs. A scoping review of available evidence from LMICs will help to understand the design elements and integration processes that have been used to thrive in an integrated care model for UHC. As no prior synthesis has been undertaken on this topic-exclusively-in the context of LMIC, scoping review is an appropriate method [23]. The methodological plasticity of scoping review will allow us to evaluate a breadth of contents-including qualitative, quantitative, mixed-method studies, reports, and other grey literature-to map, organize, identify, and report the current knowledge base from LMIC countries. This exercise will 
draw the attention of stakeholders and support in building momentum toward a systemic reform for PSI for health service delivery in India [24].

\section{Objectives}

The following three questions will guide the inquiry, analysis, and consolidation of evidence in our scoping review:

1. How are the PSI models defined during implementation in the context of LMICs?

2. What are the characteristic features of organizational and operational components of PSI models in LMIC settings?

3. What is the current evidence regarding the influence of PSI models on the health systems and population health in LMIC settings?

\section{Methods}

To conduct the scoping review, we will consider the six stages recommended by Arksey and O'Malley, and Levac et al. [25, 26]. This protocol has been registered within the Open Science Framework (Registration link: https:// osf.io/kjhzt). Besides, it is developed by following the guidance of Preferred Reporting Items for Systematic Reviews and Meta-Analyses Protocols (PRISMA-P) [27] (the see checklist is provided as Additional File 1). The result will be reported in accordance with the reporting guidance provided in the Preferred Reporting Items for Systematic Reviews and Meta-analyses extension for Scoping Reviews (PRISMA-ScR) statement [28].

\section{Stage 1: identifying the research questions}

Collaborating with researchers from Johns Hopkins Bloomberg School of Public Health (JHSPH) and Deutsche Gesellschaft für Internationale Zusammenarbeit (GIZ), India, three research questions are proposed with a broader scope while having a precise aim, listed in the previous section.

\section{Stage 2: identifying relevant literature}

The literature search will include a broad range of terms and keywords related to three concepts-"integrated care model," "primary and secondary care system," and "low- and middle-income countries." The primary source of evidence will be a structured literature search of five electronic databases (from 2000 to 2020): PubMed/MEDLINE, EMBASE, Scopus, Web of Science, and Global Index Medicus. The secondary source of potentially relevant material will be a search of the grey literature, including institutional websites (e.g., WHO and World Bank), Health Policy Plus, Google Scholar, and OpenGrey. Besides, hand-searching of the reference lists will be performed for selected articles and policy reports.
If necessary, subject matter experts and prolific authors in the field will be contacted based on the study's feasibility and necessity.

The review team will lead the design and implementation of the search strategy, and we will actively consult with a health information specialist [29]. A draft search strategy for PubMed/MEDLINE is provided in Additional File 2. After implementing the search, the title and abstracts will be downloaded, and citations will be imported into Covidence systematic review software (covidence.org). At this stage, we will remove the duplicates and organize the search records to review their titles and abstracts.

\section{Stage 3: screening and selection procedure}

We have used the "Population-Concept-Context (PCC)" framework [30] to align our study selection process with the specific research questions mentioned in the previous section. The preliminary inclusion and exclusion criteria for the screening are presented in Table 1.

The review will include a wide range of documents, such as peer-reviewed publications, methodological papers, and grey literature, published in the English language from 2000 to 2020 . However, we decided not to include chart reviews, opinion papers, case reports, editorials, and conference proceedings. Most importantly, to be included in the review, the document or article will need to include evidence on the primary-secondary integrated health care model in low- and middle-income countries, which we have considered as (see supplemental material Additional File 2 for further details):

The approach toward vertical integration of primary and secondary healthcare systems, which requires a set of coordinated strategies that involve streamlining the organizational arrangements, functional processes, service delivery apparatuses, clinical operations, and community-health facility interfaces-either by implementing independently or in any specific combinations-for incorporating secondary care functions within the primary care settings or vice versa, enabling upstream and/or downstream restructuring by augmenting health systems resources-within one setting or across health facilities-to provide evidence-based, people-centered and high-quality healthcare service and, simultaneously, to improve the performance of health systems.

Following these criteria, two independent reviewers will screen the title and the abstract (or executive summary) of the searched documents. For those eligible peer-reviewed journal articles and grey literature, which will appear to represent the subject of our scoping 
Table 1 Inclusion and exclusion criteria for the record selection process

\begin{tabular}{|c|c|c|}
\hline & Inclusion criteria & Exclusion criteria \\
\hline Concept & - Integrated primary-secondary health care model & $\begin{array}{l}\text { - Article lacking evidence or discussion on the } \\
\text { primary-secondary care integration (Example: } \\
\text { A study may report the prevalence of obesity- } \\
\text { related comorbidity and then recommending } \\
\text { an integrated primary-secondary health care } \\
\text { model as a recommendation. This will be } \\
\text { excluded during the selection process) }\end{array}$ \\
\hline Context and population & $\begin{array}{l}\text { - } 31 \text { low-income countries and } 47 \text { lower-middle- } \\
\text { income countries based on the World Bank's classification }\end{array}$ & \\
\hline Document type & $\begin{array}{l}\text { - Peer-reviewed journal articles } \\
\text { - Grey literature (such as policy brief or programmatic reports) } \\
\text { - Quantitative, qualitative, mixed or multimethod research, } \\
\text { policy analysis, methodology paper }\end{array}$ & $\begin{array}{l}\text { - Chart reviews, opinion papers, case reports, editorials } \\
\text { - Conference proceedings and posters }\end{array}$ \\
\hline Time frame & $\cdot 2000-2020$ & \\
\hline Reporting characteristics & $\begin{array}{l}\text { - Articles are written in English } \\
\text { - Complete articles that have been published }\end{array}$ & - Article not published in English \\
\hline
\end{tabular}

https://datahelpdesk.worldbank.org/knowledgebase/articles/906519-world-bank-country-and-lending-groups

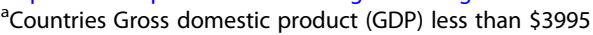

review, copies of the full documents will be obtained. Next, the reviewers will read the full document and decide to include the article/report in the review process. The research team will regularly review the result of the screening process and discuss the discrepancies. In the case of an undisputed disagreement, a senior researcher will take the role of arbitrator to resolve the issue. We acknowledge that literature search and screening is an iterative process, and we will pragmatically adapt the search criteria if necessary and reiterate the screening process [25].

\section{Stage 4: charting the data}

Full-text articles included in the scoping review will be re-appraised, and information will be charted using a data extraction form [30]. Focusing on the research question identified in stage one, we have developed a data extraction form to summarize the evidence from the document. The themes and variables included in the data extraction form are presented in Table 2, and the extended version of the data extraction form is provided in Additional File 2 as supplemental material.

Two distinct sets of information will be charted using the data extraction form. The first set of information is related to the characteristics of the article, which will include the source of the article, title, authors, publication year, country where the study was conducted, or the evidence was generated, country type (low-income or lower-middle-income), WHO region, study populations, study location (urban or rural), design and methodology of the study, etc.

The second set of information is specific to this scoping review which are, but not limited to, definition of integrated care, typologies of integration, type of service integrated, health systems building blocks integrated,
Table 2 Summary of the data extraction form to chart the evidence

\begin{tabular}{ll}
\hline Data extraction themes & Extracted data \\
\hline Study characteristics & Source \\
& Title \\
& Authors \\
& Year \\
& Country name \\
& Country type (World Bank's classification ${ }^{a}$ ) \\
& WHO region \\
& Study populations \\
& Study location \\
& Study design and methodology \\
& Definition of integrated care \\
& Typologies of integration \\
& Type of service integrated (if applicable) \\
& Health systems building blocks integrated \\
& (if applicable) \\
& Mechanism of integration (if applicable) \\
& Structure of integration (if applicable) \\
& Intensity of integration (if applicable) \\
& Organizational and operational components \\
& Success \\
& Facilitators \\
& Bottlenecks \\
& Unintended consequences \\
\hline &
\end{tabular}

https://datahelpdesk.worldbank.org/knowledgebase/articles/906519-worldbank-country-and-lending-groups

${ }^{a}$ Countries Gross domestic product (GDP) less than $\$ 3995$ 
mechanism of integration, the structure of integration, the intensity of integration, organizational and operational components of integration, success, facilitators, bottlenecks, and unintended consequences. Detailed descriptions of each of the scoping review specific thematic areas are presented in the supplementary materials of Additional File 2.

A team of two researchers will conduct the data charting process. The process will start with a pilot exercise, where the two data extractors will independently chart the data from the same five eligible articles in parallel using an initial draft of data extraction from (developed based on Table 2). A workshop will be conducted in the presence of a third senior researcher as an arbitrator to triangulate the extracted data to streamline and harmonize the data charting process between the two researchers. Next, the eligible articles will be divided among the two researchers for completing the charting process. After completing every ten articles, the senior researcher will moderate a review meeting to go over the extraction process and resolve any impending or unintended issues in the charting process. During this stage, if additional details regarding an article or report are required, we will try to reach out to the investigators for additional information while pragmatically considering the time and resources required for this process.

\section{Stage 5: collating and reporting the results}

In the fifth stage, the charted information will be summarized into thematic areas and reported in a narrative format with tables and illustrations. We will present an overview of the material included in the screening and the review process. Based on the number of articles, we will use Dedoose (dedoose.com) to extract the emergent themes and patterns from the data if necessary. The result will be clustered and presented to explore the geographic, socioeconomic, and health system variation across the countries.

\section{Stage 6: expert consultation}

While Arksey and O'Malley suggested expert consultation as optional for scoping review [25], we agree with Levac et al. [26] that this stage is essential for finalizing the scoping review. We also intend to perform a consultation exercise after developing the initial report of the scoping review. This scoping review is commissioned to inform and provide pragmatic recommendations to the $\mathrm{AB}$ program to lead a discussion for instilling PSI models as an instrument for achieving UHC. Thus, we need to organize the result and collate the evidence of our review to be palatable for the policymakers and public health practitioners in India. We will develop a roster of researchers from JHSPH, GIZ, and other organizational networks, practitioners, and public health policymakers for this consultation process to strengthen the review [31]. This is a critical component of our study as this part links the evidence with the ground reality of the Indian health system. Translating the finding of the review into pragmatic recommendations for adopting a PSI model is critical for this formative exercise.

\section{Discussion}

Ethical approval will not be necessary for the scoping review. The required information will be obtained from the publicly available literature on integrated health care models that have been adopted in LMICs, and no primary data will be collected. The investigators will also maintain a research log to record all necessary changes and methodological decisions taken while conducting the scoping review. Furthermore, the details of any changes made to the protocol of this scoping review will be outlined in the Open Science Framework, in addition to reporting in the final manuscript.

Before embarking on the journey to conduct this review, we have anticipated two operational challenges. Firstly, the three core concepts liked to the objective of this scoping review ("integrated care model," "primary and secondary care system," and "low- and middleincome countries") are relatively broad and somehow ambiguous. We anticipated that the implementation of the search strategy would produce a considerable breadth of documents, which has possible implications on the study timeframe. Secondly, as a part of the inclusion criteria, we will include studies with a wide range of methodological variation. Though this variability will not affect the title and abstract review or full-text screening process, synthesis and collation of the wide range of evidence in a harmonized way will be a challenge.

We also want to acknowledge a few anticipated limitations of this review. While structuring this scoping review, we have decided to include the five major literature databases that encompass most health sciencerelated articles. However, there is always a possibility of missing some relevant documents related to PSI models [32]. We will actively try to mitigate this limitation by exploring additional databases of the grey literature. However, unlike a systematic review, developing an allencompassing search strategy and collating all relevant studies is impossible for scoping reviews [33]. Besides, excluding the documents that are not published in English will also lead to some study attrition. Lastly, while not a limitation, it is essential to emphasize that we will not assess the quality of the evidence during the review process or compare the robustness or generalizability of different PSI models [24, 25, 30].

This scoping review forms a part of the overarching study to-explore the scope of PSI models under the $A B$ Program in India. Thus, we are particularly interested in 
building an efficient dissemination strategy for this scoping review. The dissemination process will start with sharing the initial findings of this review with other researchers, practitioners, and public health policymakers within and beyond our organizational networks as part of the consultation exercise to enhance the review's quality. The final report will be disseminated through a workshop involving critical national and state-level stakeholders for the $\mathrm{AB}$ program in India. The findings will serve as an advocacy tool for rolling out PSI for UHC in India. We will also develop publication as peerreviewed journal articles and share the result via dissemination events such as conferences and online fora.

An integrated primary, secondary, and tertiary health care model is an essential strategy to transition toward UHC. This scoping review will inform policy decisions, legislative and financing frameworks, changes in the health service organization, use of information systems, and health teams' orientation that will be needed to facilitate PSI in India. This study will serve as a foundation for modeling and implementing the efforts of PSI under the $\mathrm{AB}$ program.

\section{Supplementary Information}

The online version contains supplementary material available at https://doi. org/10.1186/s13643-020-01514-3.

\section{Additional file 1}

Additional file 2

\section{Abbreviations}

WHO: World Health Organization; UHC: Universal Health Coverage; LMICs: Low- and middle-income countries; PSI: Primary-secondary care integration; AB: Ayushman Bharat; PM-JAY: Pradhan Mantri Jan Arogya Yojana; HWCs: Health and Wellness Centres; USD: United States Dollar; NHA: National Health Authority; SHA: State Health Agency; PHCs: Primary health centers; CPHC: Comprehensive primary health care; PRISMAScR: Preferred Reporting Items for Systematic Reviews and Meta-Analyses' Extension for Scoping Reviews; JHSPH: Johns Hopkins Bloomberg School of Public Health; GIZ: Deutsche Gesellschaft für Internationale Zusammenarbeit; PRISMA-P: Preferred Reporting Items for Systematic Reviews and MetaAnalyses Protocols

\section{Acknowledgements}

We acknowledge the Deutsche Gesellschaft für Internationale Zusammenarbeit (GIZ), India, for financial support. We would also like to thank Welch Medical Library of Johns Hopkins University for providing instrumental support to conduct the scoping review. We also thank our collaborating Informationist from the Welch Medical Library, Donna Hesson, for her time and support.

\section{Authors' contributions}

$\mathrm{MH}, \mathrm{DA}$, and SG conceptualization of the thematic area of the review; $\mathrm{MH}$ and SG developed the search strategy, and $\mathrm{MH}$ conducted the preliminary searches; MH and SS together developed the first draft, and SG supervised the manuscript writing process. $\mathrm{MH}, \mathrm{SS}, \mathrm{DA}, \mathrm{SG}$, and NJ contributed to manuscript revision. All authors read and approved the read and approved the protocol for publication.

\section{Funding}

This work was supported by Deutsche Gesellschaft für Internationale Zusammenarbeit (GIZ).
Availability of data and materials

Not applicable.

\section{Ethics approval and consent to participate}

No ethical approval is required for the study.

Consent for publication

Not applicable.

\section{Competing interests}

The authors declare that there is no conflict of interest.

\section{Author details}

${ }^{1}$ Department of International Health, Johns Hopkins Bloomberg School of Public Health, Baltimore, MD, USA. ${ }^{2}$ Deutsche Gesellschaft für Internationale Zusammenarbeit (GIZ), New Delhi, India.

Received: 26 March 2020 Accepted: 27 October 2020

Published online: 09 November 2020

\section{References}

1. Lê G, Morgan R, Bestall J, Featherstone I, Veale T, Ensor T. Can service integration work for universal health coverage? Evidence from around the globe. Health Policy. 2016;120(4):406-19.

2. World Health Organization: Regional Office for Europe. Strengthening people-centred health systems in the WHO European Region: framework for action on integrated health services delivery [Internet]. Copenhagen, Denmark: World Health Organization; 2016 [cited 20203 January]. Available from: http://www.euro.who.int/_data/assets/pdf_file/0004/315787/66wd15 e FFA_HSD_160535.pdf?ua=1.

3. World Health Organization: Regional Office for Europe. Integrated care models: an overview [Internet]. Copenhagen, Denmark: WHO Regional Office for Europe; 2016 Oct [cited 20205 January] p. 42. Available from: http://www.euro.who.int/_data/assets/pdf_file/0005/322475/Integratedcare-models-overview.pdf.

4. Minkman MMN. The current state of integrated care: an overview. Journal of Integrated Care. 2012 Nov 28;20(6):346-58.

5. Lewis RQ, Rosen R, Goodwin N, Dixon J. Where next for integrated care organisations in the English NHS? [Internet]. London: The Nuffield Trust; 2010. Available from: https://www.nuffieldtrust.org.uk/files/2017-01/wherenext-integrated-care-english-nhs-web-final.pdf.

6. Smith SM, Allwright S, O'Dowd T. Effectiveness of shared care across the interface between primary and specialty care in chronic disease management. Cochrane Database Syst Rev. 2007;3:CD004910.

7. Mitchell GK, Burridge L, Zhang J, Donald M, Scott IA, Dart J, et al. Systematic review of integrated models of health care delivered at the primarysecondary interface: how effective is it and what determines effectiveness? Australian Journal of Primary Health. 2015;21(4):391.

8. Nicholson C, Jackson C, Marley J. A governance model for integrated primary/secondary care for the health-reforming first world - results of a systematic review. BMC Health Services Research [Internet]. 2013 Dec [cited 2019 Aug 25];13(1). Available from: https://bmchealthservres.biomedcentral. com/articles/10.1186/1472-6963-13-528.

9. Fusar-Poli P, Sullivan SA, Shah JL, Uhlhaas PJ. Improving the detection of individuals at clinical risk for psychosis in the community, primary and secondary care: an integrated evidence-based approach. Front Psychiatry. 2019;10:774

10. Jackson $\mathrm{CL}$, Donald M, Russell AW, Mclntyre HD. Establishing a new model of integrated primary and secondary care based around general practice: a case study of lessons learned and challenges. Aust Health Rev. 2018 Jun; 42(3):299-302.

11. Parry W, Wolters AT, Brine RJ, Steventon A. Effect of an integrated care pathway on use of primary and secondary healthcare by patients at high risk of emergency inpatient admission: a matched control cohort study in Tower Hamlets. BMJ Open. 2019 Jun;9(6):e026470.

12. National Health Authority. Ayushman Bharat [Internet]. Ayushman Bharat | Pradhan Mantri Jan Arogya Yojana. 2020 [cited 2020 Feb 6]. Available from: https://www.pmjay.gov.in/

13. National Health Authority. Pradhan Mantri Jan Arogya Yojana: Annual Report 2018-19 [Internet]. New Delhi, India; 2019 [cited 2020 Jan 2]. 
Available from: https://pmjay.gov.in/sites/default/files/2019-09/Annual\%2 OReport\%20-\%20PMJAY\%20small\%20version_1.pdf.

14. National Health Authority. About Pradhan Mantri Jan Arogya Yojana (PMJAY) [Internet]. Ayushman Bharat Pradhan Mantri Jan Arogya Yojana. 2020 [cited 2020 Jan 2]. Available from: https://www.pmjay.gov.in/about-pmjay.

15. National Health Authority. Policy \& Guidelines [Internet]. Ayushman Bharat Pradhan Mantri Jan Arogya Yojana. 2020 [cited 2020 Jan 2]. Available from: https://www.pmjay.gov.in/policy-and-guidelines.

16. Ministry of Health \& Family Welfare, Government of India. Health and Wellness Center Portal [Internet]. 2020 [cited 2020 Jan 2]. Available from: https://ab-hwc.nhp.gov.in/.

17. Ved RR, Gupta G, Singh S. India's health and wellness centres: realizing universal health coverage through comprehensive primary health care. WHO South East Asia J Public Health. 2019 Apr;8(1):18-20.

18. ENS Economic Bureau. Amartya Sen: Ayushman Bharat neglects primary healthcare, sector needs radical change [Internet]. The Indian Express. 2019 [cited 2019 Aug 25]. Available from: https://indianexpress.com/article/ business/amartya-sen-ayushman-bharat-neglects-primary-healthcare-sectorneeds-radical-change-5603964/.

19. National Health Authority. Lessons Learned in One Year implementation of PM-JAY [Internet]. New Delhi, India; 2019 [cited 2020 Jan 2]. Available from: https://www.pmjay.gov.in/sites/default/files/2019-09/Lessons\%20Learnt\%2 Osmall\%20version_0.pdf.

20. Woodman J, Lewis H, Cheung R, Gilbert R, Wijlaars LP. Integrating primary and secondary care for children and young people: sharing practice. Archives of Disease in Childhood. 2016 Sep;101(9):792-7.

21. Wakida EK, Talib ZM, Akena D, Okello ES, Kinengyere A, Mindra A, et al. Barriers and facilitators to the integration of mental health services into primary health care: a systematic review. Syst Rev. 2018;7(1):211.

22. Baxter S, Johnson M, Chambers D, Sutton A, Goyder E, Booth A. The effects of integrated care: a systematic review of UK and international evidence. BMC Health Serv Res. 2018 May 10;18(1):350.

23. The Lancet. A tiered healthcare delivery system for China. The Lancet. 2019 Mar;393(10177):1178

24. Peters MDJ, Godfrey CM, Khalil H, McInerney P, Parker D, Soares CB Guidance for conducting systematic scoping reviews. International Journal of Evidence-Based Healthcare. 2015 Sep;13(3):141-6.

25. Arksey H, O'Malley L. Scoping studies: towards a methodological framework. International Journal of Social Research Methodology. 2005 Feb;8(1):19-32

26. Levac D, Colquhoun H, O'Brien KK. Scoping studies: advancing the methodology. Implementation Science. 2010 Sep 20;5(1):69.

27. Moher D, Shamseer L, Clarke M, Ghersi D, Liberati A, Petticrew M, et al. Preferred reporting items for systematic review and meta-analysis protocols (PRISMA-P) 2015 statement. Systematic Reviews. 2015;4(1):1.

28. Tricco AC, Lillie E, Zarin W, O'Brien KK, Colquhoun H, Levac D, et al. PRISMA Extension for Scoping Reviews (PRISMA-SCR): Checklist and Explanation. Annals of Internal Medicine. 20182 October;169(7):467-73.

29. Johns Hopkins University. Informationists: Welch Medical Library [Internet]. Welch Medical Library. 2019 [cited 2019 Dec 25]. Available from: https:// welch.jhmi.edu/about/informationists

30. Micah DJ Peters, Christina Godfrey, Patricia Mclnerney, Zachary Munn, Andrea C. Tricco, Hanan Khalil. Chapter 11: Scoping reviews. In: Aromataris E, Munn Z, editors. Joanna Briggs Institute Reviewer's Manual, [Internet]. JBi; 2020. Available from: https://reviewersmanual.joannabriggs.org/.

31. Oliver $\mathrm{S}$. Marking research more useful: integrating different perspectives and different methods. In: Oliver S, Peersman G, editors. Using research for effective health promotion: McGraw-Hill Education (UK); 2001. p. 167-79.

32. Pham MT, Rajić A, Greig JD, Sargeant JM, Papadopoulos A, McEwen SA. A scoping review of scoping reviews: advancing the approach and enhancing the consistency. Res Synth Methods. 2014 Dec;5(4):371-85.

33. Gentles SJ, Lokker C, McKibbon KA. Health information technology to facilitate communication Involving Health Care Providers, Caregivers, and Pediatric patients: a scoping review. J Med Internet Res [Internet]. 2010 Jun 18 [cited 2020 Oct 22];12(2). Available from: https://www.ncbi.nlm.nih.gov/ pmc/articles/PMC2956233/.

\section{Publisher's Note}

Springer Nature remains neutral with regard to jurisdictional claims in published maps and institutional affiliations.

Ready to submit your research? Choose BMC and benefit from:

- fast, convenient online submission

- thorough peer review by experienced researchers in your field

- rapid publication on acceptance

- support for research data, including large and complex data types

- gold Open Access which fosters wider collaboration and increased citations

- maximum visibility for your research: over $100 \mathrm{M}$ website views per year

At BMC, research is always in progress.

Learn more biomedcentral.com/submissions 